\title{
Study on the Method and Strategy of the Electric Energy Meter On-site inspection Based on the Electrical information
}

\author{
Jia Li ${ }^{1}$, Wanyu Xue ${ }^{1}$, Li Gao ${ }^{1}$, Min Zhou ${ }^{1}$, Xiaoqing Huang ${ }^{1}$ and Dapeng Xing ${ }^{1}$ \\ 1State Grid Sichuan Technical Training Center, Chengdu 610072, China \\ itouvi@qq.com, xuewany@qq.com, 2425848@qq.com,348941671@qq.com,86575465@qq.com
}

Keywords: Electric Energy Meter; Field Inspection; State Assessment; Electrical Information

\begin{abstract}
In view of the shortage of frequency electric energy meter on-site inspection, a predicting evaluation method is proposed to improve the on-site inspection strategy of the electric energy meter. This method based on the data of electrical information acquisition system extracts the relevant state variables, and establishes the evaluation model, and gives the strategy of on-site inspection of electric energy meters under different conditions. A calculation example proves its validity.
\end{abstract}

\section{Introduction}

At present, the main work of electric energy metering device inspection is based on the "technology management regulation of electric energy metering device", which stipulates that the electric energy metering device in operation should be regularly inspected on the electric energy meter, and the verification methods can be divided into: first inspection, weekly inspection and sampling inspection. With the popularization and application of the intelligent electric energy meter in China, the stability and accuracy of the electric energy metering device have been greatly improved. At the same time, the power company has implemented the whole process quality supervision and management from the bidding, supply, arrival, operation and scrapping. In the past, the frequency meter has the larger workload, lower efficiency and higher safety risk. Therefore, it becomes an important problem to inspect the electric energy meters scientifically and reasonably.

At present, the electric power equipment has taken the lead in realizing the condition-based maintenance. Condition-based maintenance of power equipment can evaluate the equipment status, mainly based on the state, according to the evaluation model, and help create maintenance plan which greatly reduces the workload of on-site maintenance, and enhances the economy and safety of equipment maintenance. With the gradual establishment of the system called "San-Quan", which means full collection of electrical information, full coverage of electricity customers and full charge control of electric energy meter, it is possible to remotely on-line monitor the electric energy meter, storage electricity consumption information and evaluate the running state of meters. A series of functions such as automatic collection, load monitoring, metering and monitoring, power consumption analysis and management, line loss, remote meter reading and settlement, can be achieved by the advanced measurement infrastructure, and greatly improved the management and control means ${ }^{[1-4]}$.An on-line monitoring method based on advanced measurement infrastructure was presented by [5], but this method could not be used in engineering applications for its limitation of strict steps. The method proposed by [6-8] can detect the faults of the metering device in time and notify the treatment immediately. When the faults occur, the system developed by this method can record the load curve and help the metrological managers to find and deal with the faults earlier. However, there are still shortcomings in the practical application of the on-line monitoring system for electric energy measurement, and the monitoring capacity of the single equipment is too small.

This paper draws on the condition-based maintenance technology for electric power equipment, puts forward a method to assess the state of electric energy meters and formulate the on-site inspection strategy. Ten kinds of states are extracted from the advanced meter infrastructure, and an assessment model is established based on the normalization theory. Furthermore, a criterion for the electric energy meters stats and the strategy of on-site inspection are presented. A calculation example proves its validity. 


\section{State Parameters}

The operating state of the electric energy meters can be described from ten aspects: such as basic errors, operation error, abnormal information, operating information and so on.

The basic errors $S_{1}$ are the errors of the selected load points, which reflects the intrinsic measurement performance of the electric energy meter. The operation error $S_{2}$ is the error of the operating load, which reflects the operating measurement performance. The calculation method of error is shown in (1).

$$
\gamma=\frac{m-m_{0}}{m_{0}} \times 100 \%
$$

In (1) $\gamma$ means the error of load point, $m$ is the measured impulse, $m_{0}$ is the fixed impulse.

$$
m_{0}=\frac{C_{0} N}{C_{L} K_{I} K_{U}}
$$

In (2), $C_{0}$ is the meter constant of the standard meter, $N$ is the low frequency or high frequency pulse of the inspected meter, $C_{L}$ is the instrument constant of the inspected meter, $K_{I}$ and $K_{U}$ are the current and voltage transformer ratios accessed into the standard meter.

The abnormal information $S_{3}$ include two aspects of information which reflects the real time running situation. One is whether the electricity is abnormal; the other is the abnormal times. The operating information $S_{4}$ is the meter's operating year whose minimum value is 0.5 years.

Batch return rate $S_{5}$ refers to the proportion of batches of the three phase electric energy meters returned to their same manufacturer due to unqualified, which reflects the reputation, management and quality level of the electric energy meter manufacturer. Error dispersion $S_{6}$ refers to the standard deviation of the basic error of the same batch of qualified electric energy meters at the rated load point, which reflects the quality control of the current batch of meters. Batch operation $S_{7}$ refers to the statistical value of the operating failure rate of the same batch of power meter, reflecting the quality of the operating meter. Family defects $S_{8}$ are recognized by the common factors of design, material, technology, software and so on, which reflects the potential danger of the operating meters. The reputation of user $S_{9}$ represents whether the electric larceny happened, which affects the users' reputation. The type of user $S_{10}$ represents the type of metering device divided into five categories by the "technology management regulation of electric energy metering device", which reflects the importance of the meters.

\section{State Assessment Model of Electric Energy Meter}

The electric energy meter should be evaluated once a month. In addition, if the family defect in a class of electric energy meters has been confirmed, the overall state assessment for such electric energy meters should be carried out immediately. And if an abnormal situation happened to the electric energy meter at the time of on-line monitoring, or the on-site inspection has been finished, the state assessment should be evaluated as well.

The state assessment of the electric energy meter is composed of four sections which are the basic score $B$, test score $P$, monitoring score $M$, and family defect score $F$. The calculation method is shown in (3).

$G=B P M F$

The current state score $G$ equals to the product of $B, P, M$, and $F$. 
The Basic Score. The base score $B$ is an assessment of the current running status of the electric energy meter based on historical data.

$$
B=\sum_{i=1}^{6} B_{i}
$$

In (4), $B_{1}$ is the batch run index, $B_{2}$ is the batch error dispersion rate, $B_{3}$ is the batches operating failure index, $B_{4}$ is the basic load error index, $B_{5}$ is the operating time index and $B_{6}$ is the reputation index of user.

$$
\begin{aligned}
& B_{1}=10 \times\left(1-S_{5}\right) \\
& B_{2}=10 \times\left(1-\frac{S_{6}}{0.2 \times \gamma}\right) \\
& B_{3}=20 \times\left(1-S_{7}\right) \\
& B_{4}=10 \times \frac{\gamma-\left|S_{1-1}\right|}{\gamma}+10 \times \frac{\gamma-\left|S_{1-2}\right|}{\gamma}+10 \times \frac{\gamma-\left|S_{1-3}\right|}{\gamma} \\
& B_{5}=20-2.5 \times S_{4}
\end{aligned}
$$

$S_{1-1}$ refers to the basic error of meter at the rated voltage , rated current and the power factor: 1 . $S_{1-2}$ refers to the basic error of meter at the rated voltage, 0.05 times the rated current and the power factor: 0.5 . $S_{1-3}$ refers to the basic error of meter at the rated voltage, maximum current and the power factor: 1 .

The Test Score. The test score $P$ is the on-site inspection score of operating meter.

$$
P=\prod_{i=1}^{n} P_{i}
$$

In (9), $n$ is the latest test score. For the class III meter, $n$ equals to 3 . For the class $I I$ meter, $n$ equals to 2 . For the class I meter, $n$ equals to 1 .

$$
P_{i}=\frac{\min \left(\frac{|\gamma|-\left|S_{2}\right|}{|\gamma|}, 50 \%\right)}{50 \%} \times 100 \%
$$

The Monitoring Score. The monitoring score $M$ is the score that the on-line monitoring module discovers the abnormal operation of the electric energy meter. If the associated operating faults found in the online monitoring system have been eliminated, the effects are no longer considered.

$$
M=\left(1-S_{3}^{\prime}\right) \times \frac{10-S_{3}^{\prime \prime}}{10} \times 100 \%
$$

When the anomaly was found, $S_{3}^{\prime}$ equals to 1 . And $S_{3}^{\prime \prime}$ refers to the number of abnormal times between the two states. 
The Family Defects. In the case of family defects, the family defects score for the meter with no family defects before the family defect is eliminated is

$$
F=1-\frac{1-S_{8}}{\sqrt[n]{N}}
$$

In (12), $N$ is the total number of electric energy meters belonging to the same family. $n$ represents the number of electric energy meters that have occurred in the family, which is obtained by Table 1 .

Table 1 Reference value of $S_{8}$

\begin{tabular}{|l|c|}
\hline \multicolumn{1}{|c|}{ The status of family defects } & $\begin{array}{c}\text { Reference value } \\
\text { of } S_{8}\end{array}$ \\
\hline no significant impact and small risk & $86 \% \sim 100 \%$ \\
\hline a certain influence, but can be monitored & $51 \% \sim 85 \%$ \\
\hline a certain influence and no monitoring & $16 \% \sim 50 \%$ \\
\hline great influence on the metering performance & $0 \sim 15 \%$ \\
\hline
\end{tabular}

\section{State Determination}

From the above, the value of $\mathrm{G}$ is between $0 \sim 100$ for the normalization of each state. 100 is the best state, and 0 means that on-site inspection is needed immediately. Therefore, according to the change of the electric energy meter, the operation state of the electric energy meter is divided into four states: good, normal, attention and abnormal. The state of the electric energy meter can be evaluated as shown in Table 2, and the poor state is taken when the absolute value is not consistent with the state of the difference between the two states.

Table 2 Criterions of electric energy meter state

\begin{tabular}{|c|c|c|}
\hline $\begin{array}{c}\text { Electric energy } \\
\text { meter state }\end{array}$ & $\begin{array}{c}\text { Absolute } \\
\text { value }\end{array}$ & $\begin{array}{c}\text { The difference between the } \\
\text { last state and the present state }\end{array}$ \\
\hline Good & $100 \sim 85$ & {$[-100,5]$} \\
\hline Normal & $84 \sim 50$ & $(5,20]$ \\
\hline Attention & $49 \sim 16$ & $(20,40]$ \\
\hline Abnormal & $15 \sim 0$ & $(40,100]$ \\
\hline
\end{tabular}

\section{State Test Strategy}

Good State. The class I electric energy meter should carry out on-site inspection at least once a year in the condition that the state is "good". For the other class, the first on-site inspection can extend a cycle. And two cycles can be extended for the electric energy meter which has extended one year and keeps in a continuous "good" state.

Normal State. Pay a close attention to the electric energy meter that is evaluated as "normal", and arrange the state inspection 1/3 the normal cycle, and arrange the on-site inspection of normal cycle.

Attention State. For the electric energy meter that is evaluated as "attention", a real time state assessment should be arranged and the on-site inspection should be arranged within 3 2 months.

Abnormal State. For the electric energy meter which is evaluated as "abnormal", the field test should be carried out immediately. 


\section{Calculation Example}

The status data of a $0.2 \mathrm{~S}$ electric energy meter connected by a mutual inductor, is shown in Table 3 , which is extracted from the advanced measurement infrastructure. The electric energy meter belongs to class $\amalg$, and in the last year, its user has not stolen electricity, or destroyed the meter, etc.

Table 3 State data of the electric energy meter

\begin{tabular}{|c|c|c|c|c|c|c|c|c|c|}
\hline State & $S_{1-1}$ & $S_{1-2}$ & $S_{1-3}$ & $S_{2}$ & $S_{3}$ & $S_{4}$ & $S_{5}$ & $S_{6}$ & $S_{7}$ \\
\hline Value & 0.1 & 0.3 & 0.17 & 0.13 & 1 & 4 & 0.02 & 0.09 & 0.02 \\
\hline
\end{tabular}

According to this method, the basis score of the electric energy meter was calculated as shown in Tab 4.

Table 4 The basis score of the electric energy meter

\begin{tabular}{|c|c|l|l|l|l|l|}
\hline Index & $B_{1}$ & $B_{2}$ & $B_{3}$ & $B_{4}$ & $B_{5}$ & $B_{6}$ \\
\hline $\begin{array}{c}\text { Value(\% } \\
\text { ) }\end{array}$ & 9.8 & 1 & 19.6 & 18.6 & 10 & 10 \\
\hline
\end{tabular}

It' test score was $100 \%$, monitoring score was $90 \%$, and family defect score was $99.96 \%$. The last state score was 73.55, and the current status score was 62.08. Their difference was 11.47, therefore the state of this meter could be rated as "normal", which can be carried out on-site inspection in normal cycle, but the period of state inspection should be shortened to $1 / 3$ of normal cycle, that is, 10 days.

\section{Conclusion}

With the improvement of manufacturing technology, the stability and accuracy of electric energy meter has been greatly increased. The periodic on-site inspection is not suitable for smart meters now. Moreover, frequent on-site inspections result in waste of resources and greater security risk, so a method of state assessment for of electric energy meter has been presented on the basis of the state maintenance theory of power equipment.

This state assessment method sets up an evaluation model and make use of ten state variables coming from the advanced measurement infrastructure. At last, the criterion to divide the meter state and on-site inspection strategy have been put forward.

\section{References}

[1] Bat-Erdene B, Lee B, Kim M Y, et al. Extended smart meters-based remote detection method for illegal electricity usage[J]. Generation Transmission \& Distribution Iet, 2013, 7(11):1332-1343.

[2] Inga E, Arevalo G, Hincapié R. Optimal deployment of cellular networks for Advanced Measurement Infrastructure in Smart Grid[C]//Communications and Computing (COLCOM), 2014 IEEE Colombian Conference on. IEEE, 2014: 1-6.

[3] Wenpeng L. Advanced metering infrastructure[J]. Southern Power System Technology, 2009, 3(2): 6-10.

[4] Jiang R, Lu R, Wang Y, et al. Energy-theft detection issues for advanced metering infrastructure in smart grid[J]. Tsinghua Science and Technology, 2014, 19(2): 105-120.

[5] CHENG Y, WU H, YANG H, et al. Fuzzy Comprehensive Status Evaluation on Electric Energy Metering Device and Test Strategy Research $[\mathrm{J}][\mathrm{J}]$. Electrical Measurement \& Instrumentation, 2012, 12: 003.

[6] Bartone E J, Mendenhall Jr E L, McClutchy Jr J H, et al. System and method for monitoring and controlling energy usage: U.S. Patent 6,633,823[P]. 2003-10-14. 
[7] Ouellette M J, Hardy S G. Method and apparatus for performing the register functions for a plurality of metering devices at a common node: U.S. Patent 5,933,092[P]. 1999-8-3. 